\title{
Nosocomial Infection at the Tamale Teaching Hospital from
} 2017 To 2020

\author{
Abdallah Iddrisu Yahaya* \\ Physician, Department of Internal Medicine, Tamale Teaching Hospital, and Senior Lecturer, School of \\ Medicine, University for Development Studies, Tamale, Ghana \\ *Corresponding Author: Abdallah Iddrisu Yahaya, Physician, Department of Internal Medicine, Tamale \\ Teaching Hospital, and Senior Lecturer, School of Medicine, University for Development Studies, Tamale, \\ Ghana
}

\section{INTRODUCTION}

Historically, staphylococci, pseudomonads, and Escherichia coli have been the nosocomial infection troika; nosocomial pneumonia, surgical wound infections, and vascular accessrelated bacteremia have caused the most illness and death in hospitalized patients; and intensive care units have been the epicenters of antibiotic resistance. Acquired antimicrobial resistance is the major problem, and vancomycinresistant Staphylococcus aureus is the pathogen of greatest concern. The shift to outpatient care is leaving the most vulnerable patients in hospitals. Aging of our population and increasingly aggressive medical and surgical interventions, including implanted foreign bodies, organ transplantations, and xenotransplantation, create a cohort of particularly susceptible persons especially in developed countries. Renovation of aging hospitals increases risk of airborne fungal and other infections. To prevent and control these emerging nosocomial infections, we need to increase national surveillance, "risk adjust" infection rates so that inter hospital comparisons are valid, develop more noninvasive infection resistant devices, and work with health-care workers on better implementation of existing control measures such as hand washing.

\section{BACKGROUND}

Nosocomial infections, also known as hospitalacquired infections, are newly acquired infections that are contracted within a hospital environment. Transmission usually occurs via healthcare workers, patients, hospital equipment, or interventional procedures. The most common sites of infection are the bloodstream, lungs, urinary tract, and surgical wounds. Though any bacteria may cause a nosocomial infection, there is an increasing incidence of multidrug-resistant (MDR) pathogens causing hospital-acquired infections. This rise can be explained by indiscriminate use of antibiotics and lacking hygiene measures, especially among medical staff. Commonly seen multidrug-resistant pathogens include methicillin-resistant Staphylococcus aureus (MRSA), extended-spectrum beta-lactamaseproducing bacteria (ESBL), and vancomycinresistant enterococci (VRE). The choice of antibiotic for treating infections with these pathogens is based on the individual resistance profile and often requires additional strict isolation methods for the patient.

According to Weinstein (1998), the contribution of antibiotic resistance to excessive death rates in hospitals is difficult to evaluate, often depending on whether studies are populationbased or case-control, but evidence is mounting that antimicrobial resistance contributes to nosocomial deaths. While bacterial resistance is clearly the major threat, viral and fungal resistance could become important because of the small number of therapeutic options for these pathogens. Herpes viruses with acquired resistance to acyclovir and ganciclovir have emerged as problems, particularly in HIVinfected patients. Pathogens with intrinsic resistance often have lower pathogenicity and have disproportionately affected immunocompromised patients. For example, Candida spp. with intrinsic resistance to azole antifungal agents (e.g., C. krusei) and to amphotericin B (e.g., C. lusitaniae) have emerged as problem pathogens in oncology units. While we are facing the era of opportunists, including fungi, viruses, and parasites in immuno compromised patients, the one we fear most is the post antibiotic era. The first nosocomial inkling is MRSA with reduced susceptibility to vancomycin (7). Beyond the post antibiotic era lies the era of xenogenic infections as organs, 
transplanted from nonhuman primates, bring with them a variety of potential zoonotic pathogens. Nevertheless, traditional respiratory pathogens may yet prove to be our greatest challenge; for example, a major shift in strain type (8) could result in devastating pandemic community and nosocomial influenza A outbreaks.

\section{RESEARCH Problem}

Of late there has been increase in microbial infections in almost all departments at TTH. Most strongly hit the surgical department whereby we have post-surgical wound healing so difficult because of nosocomial infection and bacterial colonies in the wound. Some of these microbes tend to have symbiotic resistance and cause extended effect of bitter lactamase to susceptible bacteria causing resistance to antibiotics.

Internal medicine: In the internal medicine department, infectious diseases such as pneumonia, bronchitis, gastroenteritis and septic ulcers fail to heal because of lack of response to antibiotics this could be traced back to the phenomena of extended bitalactamese by bacteria such as morrasellacataris, clebsila, psyclococcus are listed as the floral collected from the respiratory tract of patients.

In the chest clinic/T.B unit patients confirmed of pulmonary tuberculosis failed to respond to the almightyisoniazide and rifampicin; which was trusted so much by the World Health Organization when it was discovered. The World Health Organization recommended that isoniaziderefamperson could clear the heart in two weeks. Patients do take the medicine for the whole course of six months but yet the signs and symptoms persist and even sputum smell continue to be positive. Research in the chest clinic from 2016-2018 confirmed that patients responded to antikoch after sputum cultures and sensitivity isolated co-infections and appropriate dose of antikoch used to treat them, this discovery prompted the rationale of this research going on now. The clinical research findings of 2016-2018 reveals the fact that coinfections, the effect of antimicrobial resistance against antikoch by micro bacterium (Abdallah Yahaya,2018).

At the paediatric department, a lot of patients had microbial resistance against bacteria. Case studies revealed the fact that icolli which was usually responding to septrin had resistance against antibiotics for a long time until cuture sensitivity showed that that specie could still respond to septrin but at attacking those, this confirms that some microbes and some antibiotics are intermediary, meaning resistant at continuing dosage but susceptible at attacking those.

In the department of obstetrics and gynaecology, nosocomial infections and co-infections have impacted on the results of induced vaginal delivery cecaerian infections. Minimal management of septic infections of all categories have been so difficult because of coinfections and beta lactamase bacterial. Nosocomial and co-infections grossly are the effects of contamination and microbial infections at the TTH obstetrics and gynaecology departments. Regular fumigation and disinfection has never been enough to stop this nosocomial infection and TTH wards, also universal precaution have never been applied or even known by some stuff until the appearance of the CORONA virus pandemic.

\section{RESEARCH QUESTIONS}

The following research questions shall be addressed by the study;

- Which nosocomial infections are emerging at Tamale Teaching Hospital (TTH)?

- Who is at risk of nosocomial infections at TTH?

- What causes nosocomial infections at TTH?

- How are nosocomial infections prevented and controlled at TTH?

\section{OBJECTIVES}

- The main objective of this study is to determine the nosocomial infections emerging at TTH.

- To determine the prevalence of various microbial colonies emerging as nosocomial infections at TTH.

- To determine how nosocomial infections are controlled and prevented at TTH.

- To determine who is at risk of nosocomial infections at TTH.

- To determine the causes of emerging nosocomial infections at TTH.

\section{LITERATURE REVIEW}

\section{Nosocomial Infections}

Nosocomial infections also referred to as healthcare-associated infections (HAI), are infections acquired during the process of receiving health care that was not present during 
the time of admission. They may occur in different areas of healthcare delivery, such as in hospitals, long-term care facilities and ambulatory settings and may also appear after discharge (Sikora and Zahra, 2020).

HAI is the most common adverse event in health care that affects patients' safety. They contribute to significant morbidity, mortality and financial burden on patients, families and healthcare systems. The emergence of multidrug resistant organisms is another complication seen with HAI. HAI affects $3.2 \%$ of all hospitalized patients in the United States, 6.5\% in the European Union/ European Economic Area and worldwide prevalence is likely much higher (Magill et al, 2018)

\section{Types of Nosocomial Infections}

Responsible pathogens originate from a variety of different sources and are represented by different sources and are represented by different types of HAI. The center for disease control and prevention broadly categorizes the types of HAI as follows:

- Central line-associated bloodstream infections (CLABSI)

- Catheter-associated urinary tract infections (CAUTI)

- Surgical site infections (SSI)

- Ventilator-associated pneumonia (VAP)

Source: Sikora and Zahra, 2020

\section{Causative Organism}

Pathogens responsible for nosocomial infections include bacteria, viruses and fungi. Specific microorganisms have unique characteristics that favor particular types of infection in susceptible hosts. The prevalence of infections caused by particular microorganisms varies depending on the healthcare facility location, healthcare setting and patient population. Overall bacteria are the most common pathogens, followed by fungi and viruses (Sikora and Zahra, 2020).

\section{Routes of Transmission}

Pathogens associated with healthcareassociated infection (HAI) may have different routes of transmission. The most common route of transmission is through contact, whereby the organisms are transmitted by direct or indirect contact. Common microorganisms that may be transmitted through contact are multidrugresistant bacteria (such as MRSA, ESBLproducing Gram-negative organisms, VRE), C. difficile and rotavirus. Droplet transmission may occur when microorganisms are transmitted from the respiratory tract by large droplets (greater than 5 microns) and travel less than 3 feet. Examples of infectious pathogens that are transmitted via the droplet route include influenza, Bordetella pertussis and Neisserria meningitides. Airborne transmission involves the transmission of organisms from the respiratory tract by small droplets (less than 5 microns) that travel long distances. Chickenpox virus, tuberculosis, measles and the novel SARSCOV-2 virus may be transmitted through the airborne route (Ferioli et al, 2020).

\section{Complications}

Complications of healthcare-associated infection are broad and depend on the type of infection, the severity of illness and implicated pathogen. The list of complications of each type of HAI can be extensive, below a few of the more common complications of each HAI are listed.

Complications of Hospital Acquired Pneumonia (HAP)/Ventilator Associated Pneumonia (VAP) include respiratory failure, empyema, parapneumonic effusions and sepsis.

Complications of Central Line-Associated Blood Stream Infection (CLABSI) include suppurative thrombophlebitis, endocarditis, septic arthritis, osteomyelitis, abscess and sepsis.

Complications of Catheter-Associated Urinary Tract Infection (CAUTI) include upper urinary tract involvement and sepsis (Sikora and Zahra, 2020).

\section{Prevention}

Hand hygiene is the most important aspect of infection control and prevention of healthcareassociated infection. Pathogenic microorganisms that are transiently on the healthcare worker are readily removed with 
routine hand hygiene and limit risk of transmission to the patient. Hand hygiene also prevents colonization and infection in the worker and the contamination of the environment. The World Health Organization has identified moments in which hand hygiene should always be practiced:

- Before touching a patient

- Before any clean or asceptic procedure

- After exposure to body fluids

- After touching a patient

- After touching patient surroundings

Source: (Mathai et al, 2010).

Alcohol-based hand sanitizers are preferred over soap and water washing except when hands are visibly soiled, contact with body fluids after using the toilet, or there is exposure to sporeforming pathogens such as $\mathrm{C}$. difficile (Mathai et al, 2010).

Studies have shown that complying to hand hygiene recommendations reduces the pathogen load, prevents transmission of HAI (Pittet et al, 2006).

Standard precautions should be practiced to protect healthcare workers. This includes the use of personal protective equipment such as gloves, gowns, masks and eye protection to protect from blood and body fluids. Transmission based precautions should be used to prevent airborne, droplet and contact transmission. A fit-tested N95 respirator should be worn and patient placement in an isolated negative pressure room to prevent airborne transmission. Surgical masks and physical distancing are precautions to prevent droplet transmission. Patient placement in a single room and healthcare worker gown and gloves are worn to avoid contact transmission of MDRO and C. difficile. Aseptic techniques should be practiced for invasive procedures and surgery.

\section{METHODOLOGY}

Cross sectional, quantitative approach of data collection

Retrospective analysis of records of patients in the clinic and records or results of sputum culture and sensitivity of the bacteriological department of the laboratory at TTH.

\section{RESULTS}

768 patients who passed through the chest clinic with pulmonary tuberculosis and other diseases were followed up. Sputum culture and sensitivity were requested, collected and sent to the bacteriolody department of our laboratory. The results were collected after 72 hours and analysed. This research continued from $1^{\text {st }}$ December,2017 to $7^{\text {th }}$ January 2019. The results were then organized into various columns and recorded as follows;

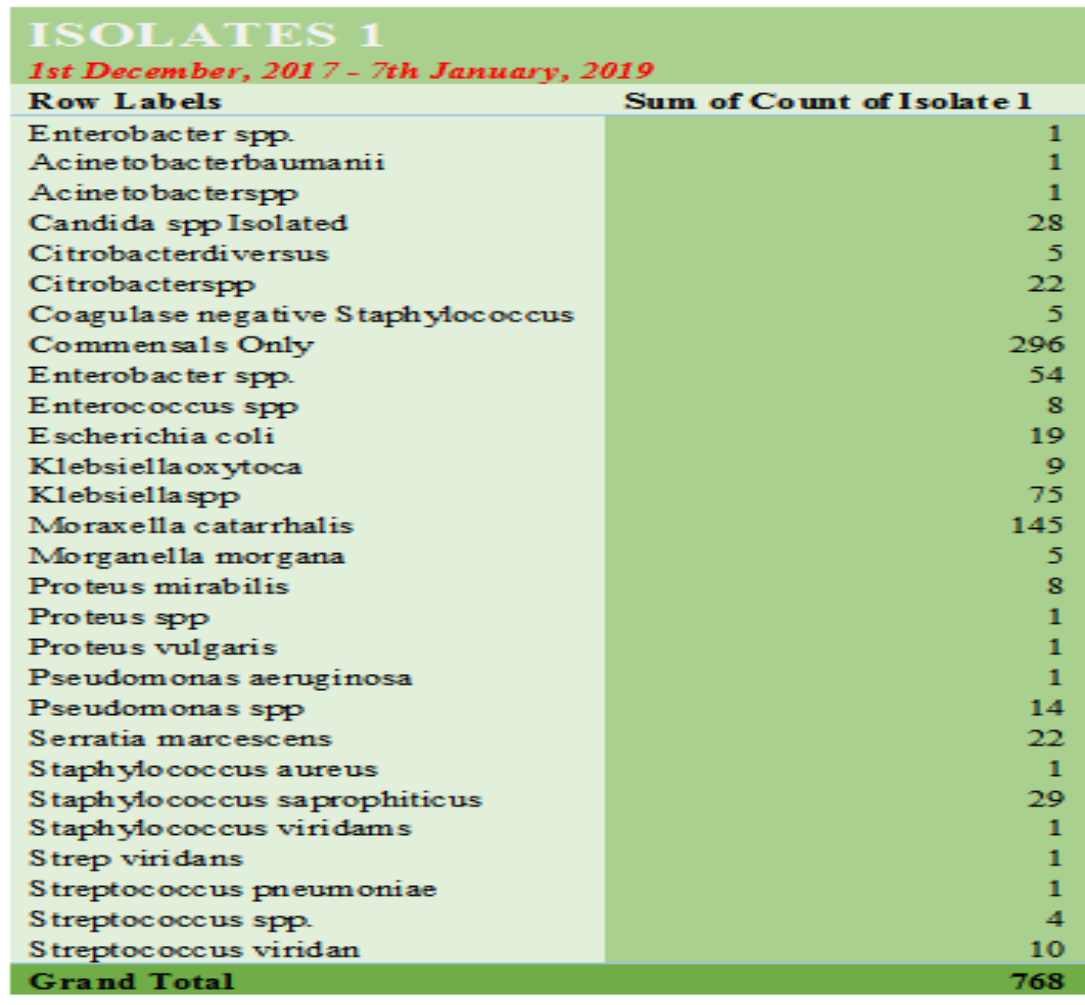

Fig1 


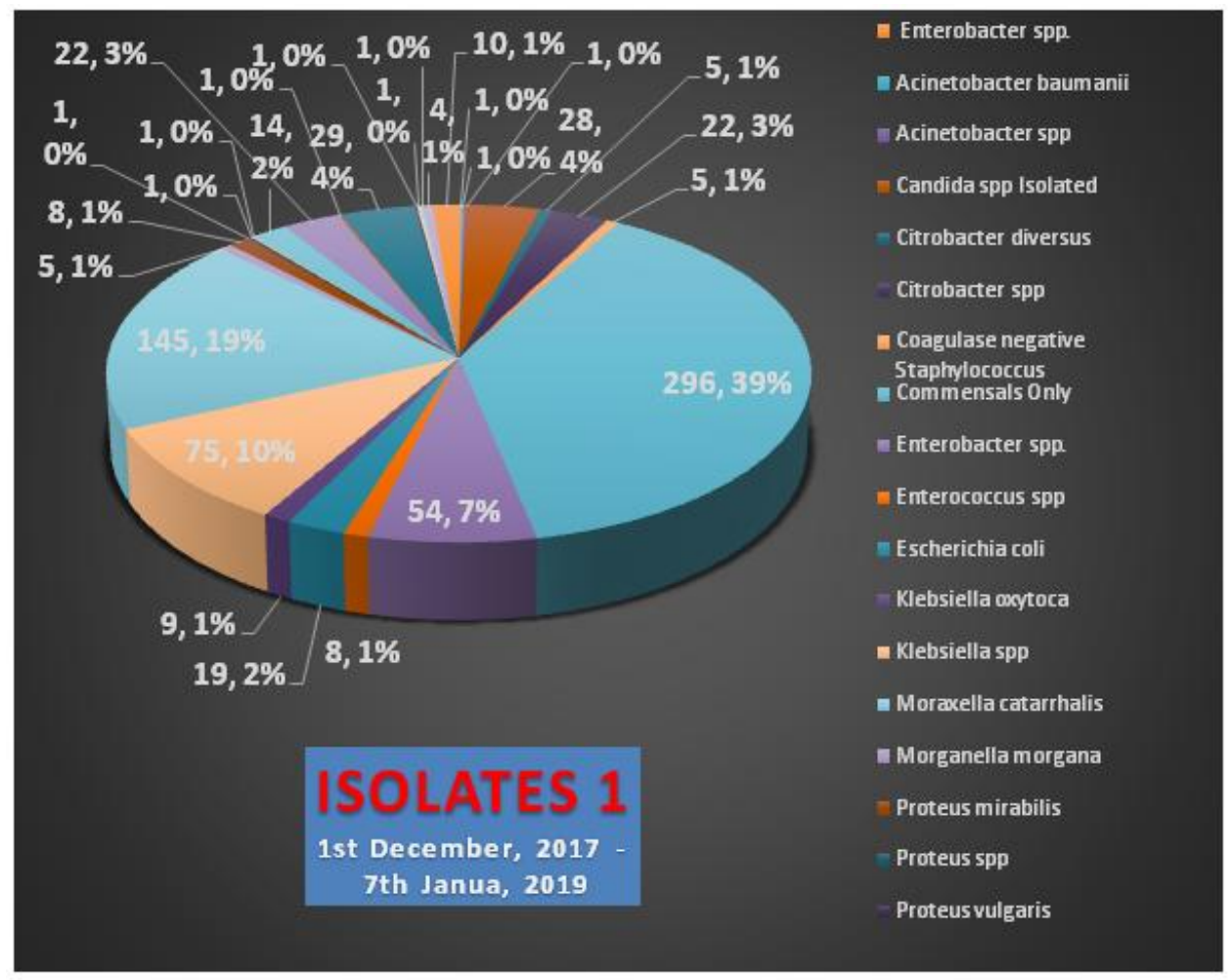

Fig 2

Table 1. Results on Nosocomial Infections Emerging at TTH Continues

\begin{tabular}{|c|c|c|}
\hline & Frequency & Percentage \\
\hline Enterobacterspp & 10 & 1.09 \\
\hline Acinetobacterbaumanii & 1 & 0.11 \\
\hline Acinetobacterspp & 1 & 0.11 \\
\hline Candida sppIsolated & 28 & 3.06 \\
\hline Citrobacterdiversus & 5 & $0.55 \%$ \\
\hline Citrobacterspp & 22 & $2.40 \%$ \\
\hline Coagulase negative Staphylococcus & 20 & $2.19 \%$ \\
\hline Commensals only & 296 & 32.35 \\
\hline Enterobacter spp. & 64 & 6.99 \\
\hline Enterococcus spp & 15 & 1.64 \\
\hline Escherichia coli & 20 & 2.19 \\
\hline Klebsiellaoxytoca & 9 & 0.98 \\
\hline Klebsiellaspp & 80 & 8.74 \\
\hline Moraxella catarrhalis & 145 & 15.85 \\
\hline Morganella morgana & 5 & 0.55 \\
\hline Proteus mirabilis & 8 & 0.87 \\
\hline Proteus spp & 15 & 1.64 \\
\hline Proteus Vulgaris & 1 & 0.11 \\
\hline Pseudomonas aeruginosa & 1 & 0.11 \\
\hline Pseudomonas spp & 20 & 2.19 \\
\hline Serratia marcescens & 22 & 2.40 \\
\hline Staphylococcus aureus & 80 & 8.74 \\
\hline Staphylococcus saprophiticus & 30 & 3.28 \\
\hline Staphylococcus viridams & 1 & 0.11 \\
\hline Strep Viridans & 1 & 0.11 \\
\hline Streptococcus pneumonia & 1 & 0.11 \\
\hline Streptococcus spp. & 4 & 0.44 \\
\hline Streptococcus Viridan & 10 & 1.09 \\
\hline Grand Total & 915 & 100 \\
\hline
\end{tabular}

Source: Bacteriology department of TTH/Chest clinic patients records book 2018-2019 


\section{Discussion OF RESUltS}

Analysing the table above, commensals were the highest (296 occupying 32.35\%). A total of 915 patients were tested, the table shows the kinds of microbes in the respiratory track of patients in the chest clinic. Some of them was nosocomial others were parasitic and some had bitter lactamase resistance extension. They are all coinfections of the respiratory track of patients at the chest clinic who were different status of pulmonary tuberculosis and other pyogenic pneumonia and bronchitis.

Looking at the table 1 it is clear that the emerging nosocomial infections are not only one group but several different colonies. For example it includes commensals, parasites, symbiotic organisms and even mixed flora. The total number of microbes documented was 915 . The bacteria included mostly Gram-positives and Gram-negatives. But the commensals had the highest prevalence occupying $32.5 \%$ (tab 1 ). The next most prevalent group of bacteria was Moraxella carrtahalis occupying $15.85 \%$. as documented in my previous research findings, Moraxella carrtahalis historically is a commensal in the respiratory tract. Mutation as a result of influence of external factors has converted the niche of Moraxella carrtahalis from commensalism to parasitic symbiant in the upper respiratory tract of patients at the chest clinic of TTH (Yahaya A.I, 2018).

The rest of the organisms found in the respiratoty tract are mainly gram positives such as Staphylococcus saprophiticus $3.28 \%$, strepviridans $0.11 \%$, streptococcus pneumonia $0.11 \%$, streptococcus spp $0.44 \%$ and streptococcus viridian $1.09 \%$. the spores of these microorganisms are left suspended in the air of the wards after sneezing or coughing of chest patients. These microbes are in their dormant forms on the dirty floors or hanging on cobwebs in the ceiling of the wards, clinics and other parts of the hospital they get inhaled by innocent patients of the ward in to their lungs or respiratory system. When sputum culture and sensitivity is done, the collected microbes in the respiratory tract are exposed in the smear microscope. Nosocomial infections are most prevalent where protocols for universal precautions are not followed; TTH is not an exception. The wards, clinics and theatres are supposed to be fumigated at least monthly. Apart from the daily cleaning and sanitizing, thorough general cleaning is supposed to be done in the hospital every week or two. These protocols of universal prevention against nosocomial infection are not followed for some time now and therefore the prevalence of nosocomial infection in TTH.

\section{CONCLUSION}

The emergence of high prevalence of nosocomial infections at TTH is as a result of failure to follow the protocol of universal prevention against nosocomial infections. For instance the World Health Organization has recommended that a health worker should wash or sanitize their hands;

- Before touching a patient

- Before any clean or asceptic procedure

- After exposure to body fluids

- After touching a patient

- After touching patient surroundings

Are these protocols practiced at TTH? No.

\section{RECOMMENDATIONS}

- Protocols for universal precaution must strictly be followed to prevent the spread of nosocomial infeactions.

- Thorough daily cleaning of the hospitals

- Fumigation of the hospital, clinics wards and theatres periodically

- As stated by the World Health Organization precautions should be followed strictly to prevent nosocomial infections.

\section{REFERENCES}

[1] Choo EJ, Chambers HF. Treatment of Methicillin-Resistant Staphylococcus aureus Bacteremia. Infection \& Chemotherapy. 2016; 48 (4): p.267. doi: 10.3947/ic.2016.48.4.267 . | Open in Read by QxMD

[2] Multi-Drug Resistant Gram Negative Bacilli (MDRGNB). http://www.hopkinsmedicine.org/ heic/infection_surveillance/mdrgnb.html.

Updated: February 12, 2017. Accessed: February 12, 2017.

[3] O'Grady NP, Alexander M, Burns LA, et al. Guidelines for the Prevention of Intravascular Catheter-related Infections. Clinical Infectious Diseases. 2011; 52 (9): p.e162-e193. doi: 10.1093/cid/cir257 . Open in Read by QxMD

[4] Shah H, Bosch W, Thompson KM, Hellinger WC. Intravascular Catheter-Related Bloodstream Infection. The Neurohospitalist. 2013; 3 (3): p.144-151. doi: 10.1177/19418744 13476043 . | Open in Read by QxMD 
[5] Rupp ME, Karnatak R. Intravascular CatheterRelated Bloodstream Infections. Infect Dis Clin North Am. 2018; 32 (4): p.765-787. doi: 10.1016/j.idc.2018.06.002 . | Open in Read by QxMD

[6] Wielders CLC, Fluit AC, Brisse S, Verhoef J, Schmitz FJ. mecA Gene Is Widely Disseminated in Staphylococcus aureus Population. J Clin Microbiol. 2002; 40 (11): p.3970-3975. doi: 10.1128/jcm.40.11.39703975.2002 . | Open in Read by QxMD

[7] New Guidelines Available on the Prevention and Control of Multi-Drug-Resistant GramNegative Bacteria in Hospitals. https://www. elsevier.com/about/press-releases/research-and -journals/new-guidelines-available-on-theprevention-and-control-of-multi-drug-resistantgram-negative-bacteria-in-hospitals. Updated:
November 18, 2015. Accessed: February 12, 2017.

[8] National and State Healthcare Associated Infections Progress Report 2016. https://www. cdc.gov/HAI/pdfs/progress-report/hai-progress -report.pdf. Updated: March 3, 2016. Accessed: February 12, 2017.

[9] WHO Guidelines on Hand Hygiene in Health Care: a Summary. http://www.who.int/gpsc/ 5may/tools/who_guidelines-handhygiene

_summary.pdf. Updated: January 1, 2009. Accessed: February 12, 2017.

[10] Inweregbu K, Dave J, Pittard A. Nosocomial infections. Continuing Education in Anaesthesia Critical Care \& Pain. 2005; 5 (1): p.14-17. doi:10.1093/bjaceaccp/mki006 . | Open in Read by QxMD

Citation: Abdallah Iddrisu Yahaya, "Nosocomial Infection at the Tamale Teaching Hospital from 2017 To 2020”, International Journal of Research Studies in Medical and Health Sciences. 2021; 6(5):1-7. DOI: https://doi.org/10.22259/ijrsmhs.0605001

Copyright: (C) 2021 Abdallah Iddrisu Yahaya. This is an open-access article distributed under the terms of the Creative Commons Attribution License, which permits unrestricted use, distribution, and reproduction in any medium, provided the original author and source are credited. 